\title{
Enhanced plasma miR-26a-5p promotes the progression of bladder cancer via targeting PTEN
}

\author{
HUI WANG ${ }^{1,2}$, ZHAO HU $^{1}$ and LI CHEN $^{3}$ \\ ${ }^{1}$ Department of Nephrology, Qilu Hospital, Shandong University, Jinan, Shandong 250014; ${ }^{2}$ Department of Nephrology, \\ The Fourth Hospital of Jinan City, Jinan, Shandong 250031; ${ }^{3}$ Department of Endocrinology, \\ Qilu Hospital, Shandong University, Jinan, Shandong 250014, P.R. China
}

Received February 5, 2018; Accepted May 22, 2018

DOI: $10.3892 / \mathrm{ol} .2018 .9163$

\begin{abstract}
The current study aimed to evaluate the expression and specific role of miR-26a-5p in the progression of bladder cancer (BC). Reverse transcription-quantitative polymerase chain reaction analysis was performed to evaluate the level of miR-26a-5p in BC cancer and healthy controls. The present data showed that plasma miR-26a-5p was significantly increased in BC patients. Furthermore, BC tissues exhibited greater levels of miR-26a-5p compared with adjacent non-neoplastic tissues-26a-5p. Compared with BC patients at Ta-T1 stage, the level of miR-26a-5p was significantly elevated in BC patients $\geq \mathrm{T} 2$. BC patients at G3 stage demonstrated a higher plasma miR-26a-5p level compared with those at G1/2 stage. Receiver operating characteristic (ROC) analysis indicated that miR-26a-5p could differentiate BC patients from controls. Additionally, Kaplan-Meier analysis demonstrated that plasma miR-26a-5p negatively correlated with survival of BC patients. Dual luciferase reporter assay indicated that miR-26a-5p significantly suppressed the relative luciferase activity of pmirGLO-PTEN-3'UTR compared with the control. In conclusion, the current study indicated novel data that the levels of plasma miR-26a-5p was significantly increased in BC patients. Furthermore, the present study suggested that determination of plasma miR-26a-5p level could help to distinguish BC patients from healthy controls via targeting PTEN.
\end{abstract}

\section{Introduction}

Bladder cancer (BC) is one of the most common urinary system malignancies worldwide (1). Reports indicate that the incidence and mortality of $\mathrm{BC}$ continues to increase in recent years (2). Furthermore, there is an approximately 3:1 male

Correspondence to: Dr Li Chen, Department of Endocrinology, Qilu Hospital, Shandong University, 44 Wenhua Xi Road, Jinan, Shandong 250014, P.R. China

E-mail: qiluhospital0531@yeah.net

Key words: miR-26a-5p, bladder cancer, PTEN, biomarker to female ratio among BC patients. Current studies suggest multiple signaling pathways are involved in BC progression $(3,4)$, however, specific mechanisms of BC etiology are still unknown. Due to invasive and costly procedures such as cystoscopy, researchers are exploring potential biomarkers from either urine or peripheral blood $(5,6)$. Consequently, investigation of novel noninvasive biomarkers to enhance the early detection of BC is of great importance.

MicroRNAs (miRNAs) are small non-coding RNAs with approximately 22 nucleotides, which are extensively involved in multiple biological processes including cell proliferation, differentiation and apoptosis (7,8). Abnormal expression of miRNAs has been widely identified in different diseases $(9,10)$. As one example, lower expression of miR-125b was found in BC tissues as compared to adjacent normal tissues (11). Conversely, miR-451 is shown to suppress BC cell invasion and proliferation via modulating EMT (12). Additionally, several differentially expressed miRNAs are found to be useful for the diagnosis of BC patients (13-15). For instance, plasma miR-497 and miR-663b the levels of the miRs could help to distinguish both groups, including BC patients and healthy control (13).

In the present study, we focused on miR-26a-5p, a miRNA reported to be dysregulated in various tumors including melanocyte transformation and hepatocarcinoma $(16,17)$. Little is known about the specific role of miR-26a-5p in the progression of BC. Here, we aim to evaluate the expression of miR-26a-5p in $\mathrm{BC}$ tissues and the potential underlying mechanism.

\section{Materials and methods}

Cell culture and transfections. The human BC cell lines (J82, HT1376, RT4, T24 and TCCSUP), immortalized human bladder epithelium (HCV29) cell and 293 cells were purchased from American Type Culture Collection (ATCC, Manassas, VA, USA). All cell lines were cultured in Dulbecco's modified Eagle's medium/F12 (DMEM) (Invitrogen; Thermo Fisher Scientific, Inc., Waltham, MA, USA) supplemented with $10 \%$ heat-inactivated fetal calf serum (Gibco; Thermo Fisher Scientific, Inc.), $100 \mathrm{U} / \mathrm{ml}$ penicillin and streptomycin in $25-\mathrm{cm}^{2}$ culture flasks at $37^{\circ} \mathrm{C}$ in a humidified atmosphere with $5 \% \mathrm{CO}_{2}$.

Patients and specimens. The human clinical samples were obtained from surgical specimens from a total of 100 patients 
with BC at Qilu Hospital (Jinan, China). The corresponding adjacent non-neoplastic tissues from the macroscopic tumor margin were isolated at the same time and used as controls. The details for patients' information were included in Table I.

All samples were immediately snap frozen in liquid nitrogen and stored at $-80^{\circ} \mathrm{C}$ until RNA extraction. Whole blood samples were prospectively collected from $\mathrm{BC}$ patients and control patients without urologic malignancies. Whole blood $(5-8 \mathrm{ml})$ was collected in an ethylene diamine tetraacetic acid (EDTA) tube. The sample was centrifuged twice at $4^{\circ} \mathrm{C}$. Plasma (supernatant after second centrifugation) was then stored at $-80^{\circ} \mathrm{C}$. The research protocols were approved by Qilu Hospital (Jinan, China), and written informed consent was obtained from the participants.

Plasma RNA Isolation. Total RNA was isolated with RNAVzol LS (Vigorous, Beijing, China) according to the specific manufacturer instructions to isolate small RNAs. Quality, quantity and integrity of RNA were monitored using a NanoDrop spectrophotometer (ND-1000; Nanodrop Technologies; Thermo Fisher Scientific, Inc.).

$R T-q P C R$ validation. RNA was reverse transcribed into cDNA using the Prime-Script one-step RT-qPCR kit (C28025-032; Invitrogen; Thermo Fisher Scientific, Inc.). Detailed RT-qPCR procedure was described as follows: $95^{\circ} \mathrm{C}$ for $10 \mathrm{~min}$ followed by 50 cycles of $95^{\circ} \mathrm{C}$ for $10 \mathrm{sec}, 55^{\circ} \mathrm{C}$ for $10 \mathrm{sec}, 72^{\circ} \mathrm{C}$ for $5 \mathrm{sec} ; 99^{\circ} \mathrm{C}$ for $1 \mathrm{sec} ; 59^{\circ} \mathrm{C}$ for $15 \mathrm{sec} ; 95^{\circ} \mathrm{C}$ for $1 \mathrm{sec}$; and then cooling to $40^{\circ} \mathrm{C}$. U6 was used as an internal control. The relative expression levels were calculated with the $2^{-\Delta \Delta \mathrm{Ct}}$ method, and experiments were repeated in triplicate.

Transient transfection. A total of $6 \times 10^{5}$ cells were equally seeded in the 6-well plates with $2 \mathrm{ml}$ DMEM culture medium containing serum and antibiotics. miR-26a-5p mimic, inhibitor, miR negative control (5'-UUCUCCGAACGUGUC ACGUTT-3'), were mixed with HiperFect transfection reagent (Qiagen GmbH, Hilden, Germany) and incubated at room temperature for $10 \mathrm{~min}$. The complex was then transfected into HT1376 cells for $48 \mathrm{~h}$.

Cell viability analysis. To examine cell viability, HT1376 cells were seeded in 96-well plates at a density of $1.0 \times 10^{4}$ cells/per well.miR-26a-5p mimics, inhibitors or a scramble/non-targeting oligo negative control (NC) were transfected into cells at $24,48,72 \mathrm{~h}$ after seeding of cells. MTT assay was performed as previously described (18).

Western blot analysis. HT1376 cells were treated with radioimmunoprecipitation assay buffer (Beijing Solarbio Science \& Technology Co., Ltd., Beijing, China) containing $1 \%$ (v/v) phenylmethylsulfonyl fluoride (Beijing Solarbio Science \& Technology Co., Ltd.), 0.3\% (v/v) protease inhibitor (Sigma-Aldrich; Merck KGaA) and $0.1 \%$ (v/v) phosphorylated proteinase inhibitor (Sigma-Aldrich; Merck KGaA, Darmstadt, Germany). A BCA protein assay kit (Pierce; Thermo Fisher Scientific, Inc.) was used to determine the protein concentration. Subsequently, supernatants were extracted from the lysates following centrifugation at $11,000 \mathrm{x}$ g at $4^{\circ} \mathrm{C}$ for $15 \mathrm{~min}$. Equal amounts of protein
(30 $\mu \mathrm{g} /$ lane) were separated using 10\% SDS-PAGE at $300 \mathrm{~mA}$ for $2 \mathrm{~h}$ and transferred onto a polyvinylidene fluoride membrane, as previously reported (10). Nonspecific binding was blocked using $8 \%(\mathrm{w} / \mathrm{v})$ milk in Tris-buffered saline with Tween 20 (TBS-T) for $2 \mathrm{~h}$ at room temperature. The following primary antibodies were used: GAPDH (cat no. 5174), PTEN (cat no. 9188) all 1:1,000 dilution; Cell Signaling Technology, Inc., (Danvers, MA, USA). Following several washes with Tris-buffered saline with Tween 20, the membranes were incubated with horseradish peroxidase-conjugated goat anti-rabbit IgG (1:5,000; ZB-2306; Zhongshan Gold Bridge Biological Technology Co., Beijing, China) for $2 \mathrm{~h}$ at room temperature and then washed with TBS-T. Proteins were detected using enhanced chemiluminescence RapidStep ${ }^{\mathrm{TM}}$ ECL, according to the manufacturer's protocol (cat. no. 345818, Merck KGaA). ImageJ v.1.8.0 (National Institutes of Health, Bethesda, MD, USA) was applied to quantify the relative protein levels. GAPDH was used as an internal control.

miRNA target prediction and dual-luciferase reporter assay. TargetScan (https://www.targetscan.org) was applied to determine the potential target gene of miR-26a-5p. The $3^{\prime}$ untranslated region (3'UTR) of PTEN was cloned into the pmirGLO plasmid. For pmirGLO-PTEN-3'UTR-1, the fragment including the position 41-47 and position 1261-1268 of PTEN 3'UTR, was cloned into dual luciferase reporter vector pmirGLO. For pmirGLO-PTEN-3'UTR-2, the fragment including position 2619-26a-5p26 of PTEN 3'UTR was cloned into dual luciferase reporter vector pmirGLO. The primers used to amplify PTEN-3'UTR-1 and PTEN-3'UTR-2 were listed as follows: PTEN-3'UTR-1 forward: ATCAAGAGG GATAAAACAC; PTEN-3'UTR-1 reverse: CTTTTTTTTGTG CAGCATTG; PTEN-3'UTR-2 forward: GACACGTTTTCC ATACCTTGTCA; PTEN-3'UTR-1 reverse: GGCAACCAC AGCCATCGTTA. The amplification was performed using a TrasnDirect Animal Tissue PCR kit (Beijing Transgen Biotech Co., Ltd., Beijing, China). In brief, $4 \mu 1$ unpurified lysate was mixed with $0.4 \mu \mathrm{l}$ forward primer, $0.4 \mu \mathrm{l}$ reverse primer, $10 \mu \mathrm{l}$ 2xTransDirect PCR SuperMix, and $5.2 \mu \mathrm{lddH} 2 \mathrm{O}$. The PCR conditions were $94^{\circ} \mathrm{C}$ for $10 \mathrm{~min}$, followed by 35 cycles of $94^{\circ} \mathrm{C}$ for $30 \mathrm{sec}, 55^{\circ} \mathrm{C}$ for $30 \mathrm{sec}, 72^{\circ} \mathrm{C}$ for $1 \mathrm{~min}$, and a final extension step of $72^{\circ} \mathrm{C}$ for $5 \mathrm{~min}$. The pmirGLO promoter vector and amplified fragments were digested by XhoI and $\mathrm{KpnI}$ and purified by $2 \%$ agarose gel electrophoresis. The digested fragment was then inserted into the pmirGLO vector upstream of the simian vacuolating virus 40 promoter and the pmirGLO-PTEN-3'UTR-1 or pmirGLO-PTEN-3'UTR-2 was successfully established.

After $293 \mathrm{~T}$ cells were seeded for $24 \mathrm{~h}$, miR-26a-5p or scramble were cotransfected with blank pmirGLO or pmirGLO-PTEN-3'UTR-1 or pmirGLO-PTEN-3'UTR-2 using vigofect (Vigorous) according to the instructions. The luciferase activity was analyzed with the Dual-Luciferase Reporter Assay System (E1910; Promega Corporation, Madison, WI, USA).

Statistics. The data are represented as the mean \pm standard error (SD). The two-tailed unpaired student's t-tests were used for comparisons of two groups, including Table II.Comparisons of means among multiple groups were determined using 
Table I. Clinicopathological features of patients with BC and healthy controls.

\begin{tabular}{lcc}
\hline Variables & BC patients & Healthy controls \\
\hline Sex & & \\
Female & 25 & 11 \\
Male & 75 & 39 \\
Age & & \\
$\geq 60$ & 64 & 35 \\
$<60$ & 36 & 15 \\
Stage & & - \\
Ta & 33 & - \\
T1 & 27 & - \\
T2 & 17 & - \\
T3 & 13 & - \\
T4 & 10 & - \\
Grade & & - \\
1 & 32 & \\
2 & 38 & \\
3 & 30 & \\
\hline B & &
\end{tabular}

$\mathrm{BC}$, bladder cancer.

one-way analysis of variance followed by Tukey's post hoc test. Receiver operating characteristic curve (ROC) analysis was used to assess miR-26 as a biomarker, and the Area Under the Curve (AUC) was reported (IBM SPSS v.20.0; IBM Corp., Armonk, NY, USA). Spearman's correlation coefficient was used to test the correlation between the expression of miR-26 and clinical index (IBM SPSS v.20.0; IBM Corp.). $\mathrm{P}<0.05$ was considered to indicate a statistically significant difference.

\section{Results}

Increased miR-26a-5p level in the plasma and tissues of $B C$ patients. First, we explored the relative level of miR-26a-5p in the plasma and tissues of BC patients and healthy individuals. Plasma miR-26a-5p showed significantly elevated levels in BC patients $(33.7 \pm 13.5)$ vs. healthy controls $(1 \pm 0.87)-26 a-5 p$ (Fig. 1A). Meanwhile, we also evaluated the relative level of miR-26a-5p in $\mathrm{BC}$ patients and healthy control. As shown in Fig. 1B, the relative expression of miR-26a-5p was much higher in BC tissues of BC patients $(7.45 \pm 1.23)$ than that of adjacent non-malignant tissue

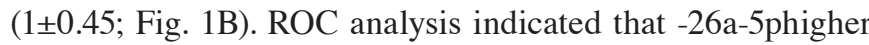
levels of plasma miR-26a-5p could help to distinguish both groups, with an ROC curve areas of 0.935 (95\% confidence interval: 0.817-1.000; $\mathrm{P}<0.0001$; Fig. $1 \mathrm{C})$. And the diagnosis was relatively more accurate.

miR-26a-5p positively correlated with BC metastasis and invasion level. The relationship between miR-26a-5p expression and different clinicopathological factors is demonstrated in Table II. Increased miR-26a-5p expression in the plasma of $\mathrm{BC}$ patients was found to be significantly associated with tumor grade (Ta-T1 vs. $\geq \mathrm{T} 2$ : $1 \pm 0.68$ vs. $21.5 \pm 5.93$,
Table II. Association between miR-26a-5p expression and different clinicopathological features of patients with BC.

\begin{tabular}{|c|c|c|c|}
\hline Variables & No. & $\begin{array}{c}\text { Relative } \\
\text { miR-26a-5p level }\end{array}$ & P-value \\
\hline \multicolumn{4}{|l|}{ Sex } \\
\hline Female & 25 & $1 \pm 1.12$ & \\
\hline Male & 75 & $1.65 \pm 0.23$ & 0.73 \\
\hline \multicolumn{4}{|l|}{ Age (years) } \\
\hline$<60$ & 36 & $1 \pm 2.12$ & \\
\hline$\geq 60$ & 64 & $1.34 \pm 0.78$ & 0.26 \\
\hline \multicolumn{4}{|c|}{ No. of tumors } \\
\hline Single & 47 & $1 \pm 1.56$ & \\
\hline Multiple & 53 & $0.96 \pm 1.21$ & 0.48 \\
\hline \multicolumn{4}{|l|}{ Stage } \\
\hline Ta-T1 & 60 & $1 \pm 0.68$ & \\
\hline$\geq \mathrm{T} 2$ & 40 & $21.5 \pm 5.93$ & 0.00043 \\
\hline \multicolumn{4}{|l|}{ Grade } \\
\hline $\mathrm{G} 1 / 2$ & 70 & $1 \pm 0.67$ & \\
\hline G3 & 30 & $17.8 \pm 4.56$ & 0.0054 \\
\hline
\end{tabular}

$\mathrm{P}=0.00043$; Fig. 2A) and tumor stage (G1/G2 vs. G3: $1 \pm 0.67$ vs. $17.8 \pm 4.56, \mathrm{P}=0.0054$; Fig. $2 \mathrm{~B}$ ). However, no significant correlation was observed between miR-26a-5p expression and other clinicopathologic variables such as age, gender, tumor size, and number of tumors (all $\mathrm{P}>0.05$; Table II).

Furthermore, we also compared the levels of miR-26a-5p in the human BC cell lines (J82, HT1376, RT4, T24 and TCCSUP) and immortalized human bladder epithelium (HCV29) cell. As shown in Fig. 2C, the relative level of miR-26a-5p was highest in HT1376 cells (7.45 \pm 0.57$)$, followed by T24 (5.14 \pm 0.74$)$, J82 (3.24 \pm 0.42$)$, RT4 (2.76 \pm 0.38$)$, and TCCSUP (1.97 \pm 0.34$)$ cells, compared with that of HCV29 $(1 \pm 0.22)$ cells. For the subsequent in vitro assay, HT1376 cells were further used.

Plasma miR-26a-5p negatively correlated with survival of $B C$ patients. To examine whether plasma miR-26a-5p could predict the prognosis of BC, Kaplan-Meier analysis was carried out. Our data showed that patients with higher plasma levels of the miR-26a-5p who had worse survival rate-26a-5p than those with lower plasma miR-26a-5p level, with 5-year overall survival rates of $27.86 \%$ and $51.46 \%$, respectively (Fig. 3).

PTEN was a target gene of miR-26a-5p. Based on TargetScan analysis, a conserved binding site of miR-26a-5p in the 3'UTR of PTEN was identified (Fig. 4A). Dual luciferase reporter assay indicated that miR-26a-5p significantly suppressed the relative luciferase activity of pmirGLO-PTEN-3'UTR vs. that of control in 293 cells (Fig. 4B). Western blot analysis also showed that overexpression of miR-26a-5p suppressed the expression of PTEN in HT1376 cells (Fig. 4C), while inhibition of miR-26a-5p increased the protein level of PTEN in HT1376 cells (Fig. 4D). These data indicated that PTEN was a target gene of miR-26a-5p. 
A

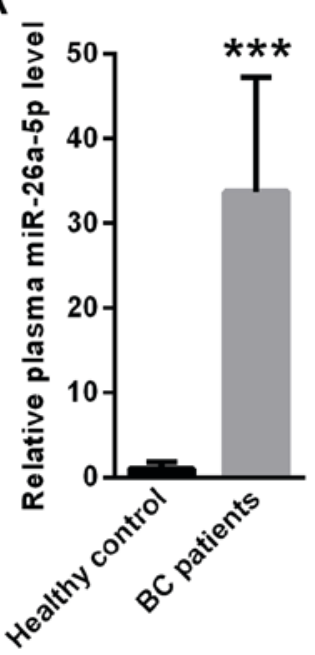

B

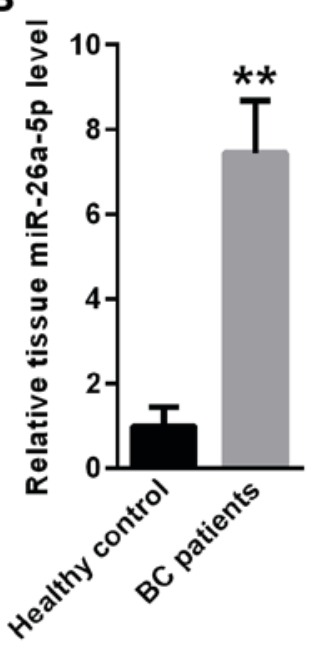

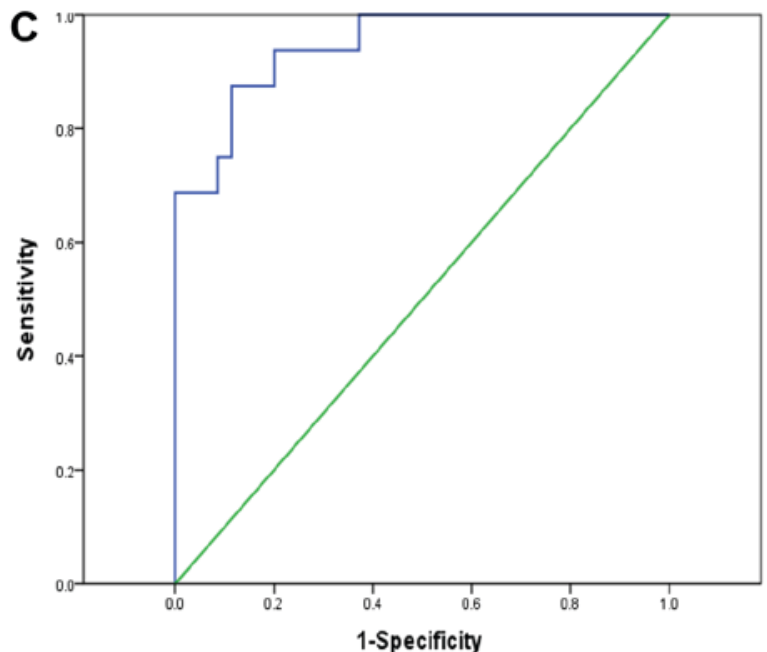

Figure 1. Expression of miR-26a-5p in the plasma and tissues of BC patients and healthy individuals. (A) Compared with healthy individuals, plasma miR-26a-5p was significantly enhanced. (B) The expression of miR-26a-5p was much higher in bladder cancer tissues of BC patients than that of healthy controls. (C) ROC analysis indicated that determination of plasma miR-26a-5p level could help to distinguish both groups-26a-5p. ${ }^{* *} \mathrm{P}<0.01,{ }^{* * * *} \mathrm{P}<0.001$ vs. control. BC, bladder cancer.
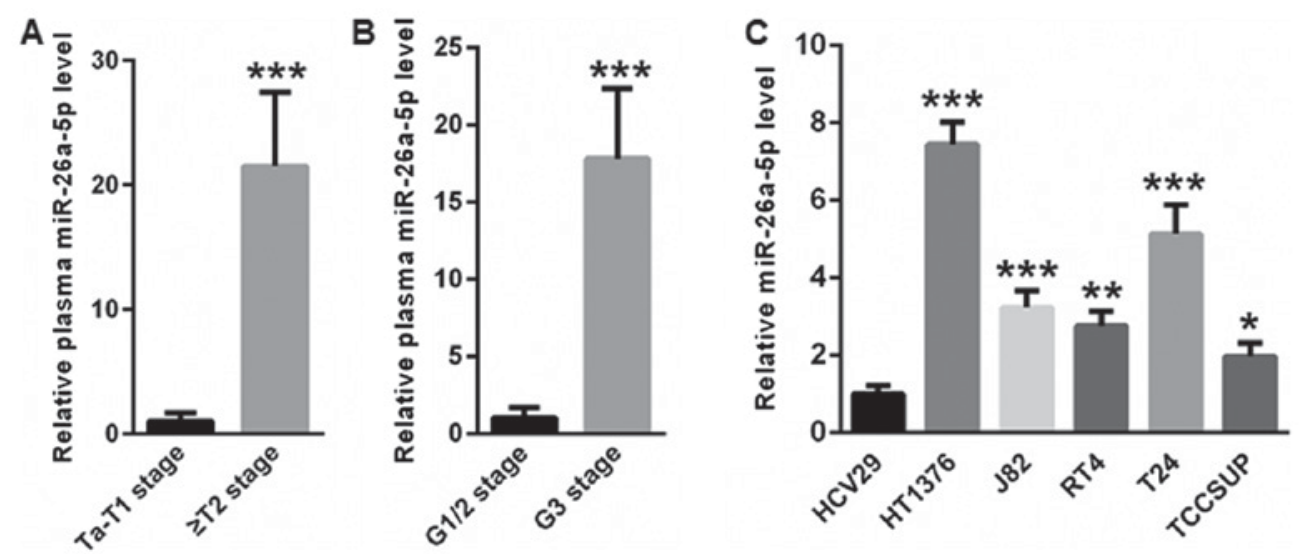

Figure 2. miR-26a-5p positively correlated with BC metastasis and invasion level. (A) Compared with BC patients at Ta-T1 stage, the level of miR-26a-5p was significantly enhanced in BC patients. (B) BC patients at G3 stage demonstrated much higher plasma miR-26a-5p level than those at G1/2 stage. (C) The relative level of miR-26a-5p was highest in HT1376 cells, followed by T24, J82, RT4, and TCCSUP cells, compared with that of HCV29 cells. $\mathrm{P}<0.05,{ }^{* *} \mathrm{P}<0.01,{ }^{* * *} \mathrm{P}<0.001$ vs. control. BC, bladder cancer.

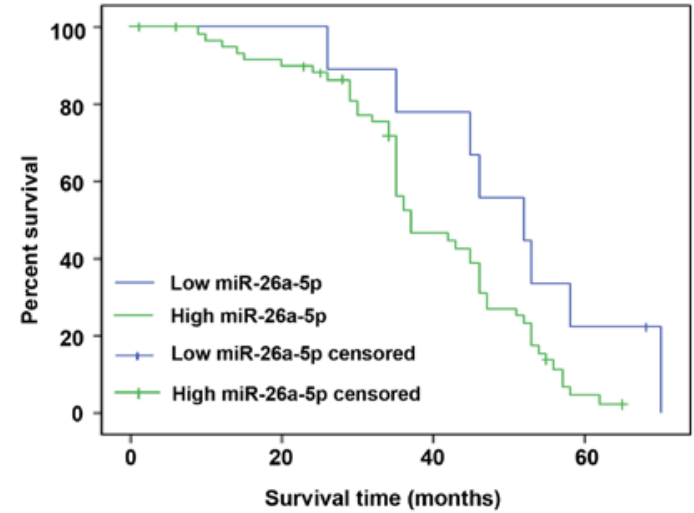

Figure 3. Kaplan-Meier analysis showed that plasma miR-26a-5p negatively correlated with survival of bladder cancer patients.

\section{Discussion}

$\mathrm{BC}$ is a major urinary malignancy around the world $(19,20)$. It is reported that chromosomal anomalies, genetic polymorphisms, and genetic and epigenetic alterations may contribute to the tumorigenesis and progression of $\mathrm{BC}(21)$. Therefore, the early detection of $\mathrm{BC}$ is important. At present, increasing evidence has suggested that miRNAs may be potential biomarkers for the diagnosis and therapy targets of $\mathrm{BC}$ patients.

miRNAs can stably exist in body fluids including serum, plasma, saliva, urine and tears $(22,23)$. Furthermore, miRNAs can be easily detected in small amounts in samples and are resistant against degradation (24). These characteristics of miRNAs render them attractive as potential biomarkers $(8,25)$. In the present study, we detected the level of miR-26a-5p in $\mathrm{BC}$ patients and our data showed that plasma miR-26a-5p was significantly increased in BC patients. Furthermore, miR-26a-5p expression was also elevated in the BC tissues compared with adjacent non-neoplastic tissues. Further analysis showed positive correlation between miR-26a-5p and clinical outcome. Compared with BC patients at Ta-T1 stage, the level of miR-26a-5p was significantly enhanced in $\mathrm{BC}$ patients $\geq \mathrm{T} 2$. Furthermore, BC patients at G3 stage 
A

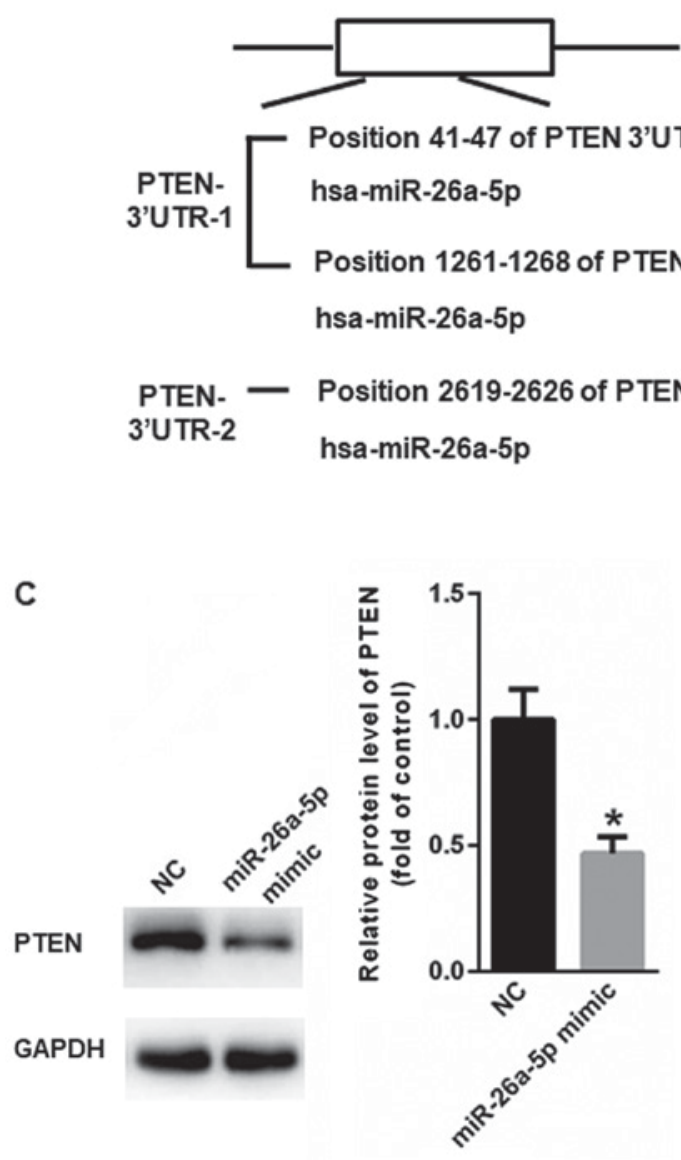

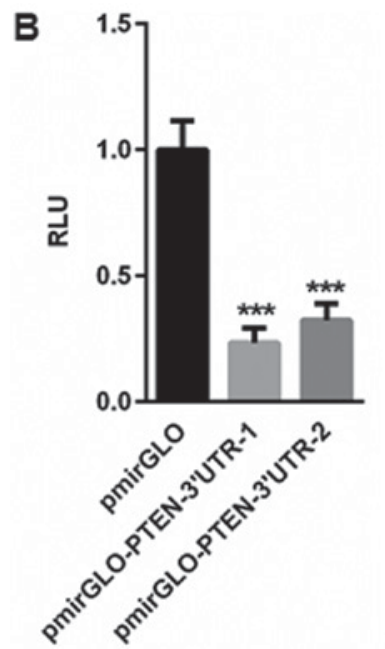

D

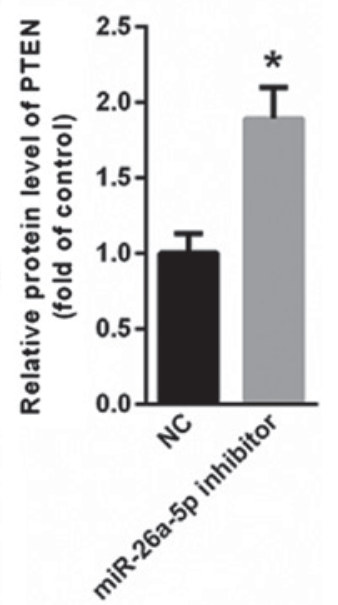

Figure 4. PTEN was a target gene of miR-26a-5p. (A) A conserved binding site of miR-26a-5p in the 3'UTR of PTEN was identified. (B) Dual luciferase reporter assay indicated that miR-26a-5p significantly suppressed the relative luciferase activity of pmirGLO-PTEN-3'UTR as opposed to the activity seen with the control in 293 cells. (C) Western blot analysis also showed that overexpression of miR-26a-5p suppressed the expression of PTEN, while inhibition of miR-26a-5p increased the protein level of PTEN in HT1376 cells (D). RLU: Relative luciferase units. ${ }^{*} \mathrm{P}<0.05$, ${ }^{* * * *} \mathrm{P}<0.001$ vs. control.

demonstrated much higher plasma miR-26a-5p level than those at G1/2 stage. These data showed that miR-26a-5p positively correlated with therapy and outcome. ROC analysis indicated miR-26a-5p could differentiate BC patients from healthy controls. Additionally, Kaplan-Meier analysis demonstrated that plasma miR-26a-5p negatively correlated with survival of $\mathrm{BC}$ patients. These data indicated that plasma miR-26a-5p may be useful for the early detection of cancer and individualized therapies.

The above observations led us to further explore the underlying mechanism by which miR-26a-5p regulates the progression and development of BC. Our data showed that PTEN was a target gene of miR-26a-5p. PTEN is widely acknowledged as a tumor suppressor that is mutated in multiple tumors (26). In the progression of $\mathrm{BC}$, reduction of PTEN could activate the AKT/PKB signaling pathway, thereby increasing cancer cell growth and survival $(27,28)$. Our data indicated that miR-26a-5p may suppress the expression of PTEN thereby resulting in the malignant proliferation and viability of cancer cells.

In certain patients with advanced BC, loss of PTEN activity is observed (29). PTEN is a tumor suppressor gene that negatively regulates cell proliferation and survival and reduction of PTEN induces constitutive phosphorylation of the AKT/PKB signaling pathway, thereby enhancing cancer cell cycle progression, survival and migration in BC patients (26). However, more direct functional studies are required to prove this pathologic role of miR-26a-5p in the progression of BC.

In summary, the present study showed novel data that the levels of plasma miR-26a-5p was significantly increased in BC patients. Furthermore, the present study showed determination of plasma levels of miR-26a-5p could help to distinguish both groups-26a-5p via targeting PTEN. However, many limitations still exist in the present study. First, large samples are still required to further validate the oncogenic role of miR-26a-5p. Second, it is necessary to further explore the regulation of the malignant phenotypes of $\mathrm{BC}$ cells via in vitro ectopic expression or loss of function study on miR-26a-5p. Lastly, it is interesting to evaluate the antago-miR-based silencing of miR-26a-5p in clinical application due to the significant upregulation of miR-miR-26a-5p in plasma of $\mathrm{BC}$ patients.

\section{Acknowledgements}

Not applicable. 


\section{Funding}

The present study was supported by a grant from Qilu Hospital, Jinan, China, (grant no. SDUQL-20160708).

\section{Availability of data and materials}

The datasets used and/or analyzed during the current study are available from the corresponding author on reasonable request.

\section{Authors' contributions}

HW performed the experiments and analyzed the data. $\mathrm{ZH}$ performed part of the RT-qPCR experiments. LC designed the experiments, analyzed the data and gave final approval of the version to be published. All authors read and approved the final manuscript.

\section{Ethics approval and consent to participate}

The research protocols were approved by Qilu Hospital (Jinan, China) and written informed consent was obtained from the participants.

\section{Consent for publication}

Informed consent for participation in the present study or use of their tissue was obtained from all participants.

\section{Competing interests}

The authors declare that they have no competing interests.

\section{References}

1. Torre LA, Bray F, Siegel RL, Ferlay J, Lortet-Tieulent J and Jemal A: Global cancer statistics, 2012. CA Cancer J Clin 65 87-108, 2015

2. Siegel R, Naishadham D and Jemal A: Cancer statistics, 2013 CA Cancer J Clin 63: 11-30, 2013.

3. Latini DM, Lerner SP, Wade SW, Lee DW and Quale DZ: Bladder cancer detection, treatment and outcomes: Opportunities and challenges. Urology 75: 334-339, 2010.

4. Lokeshwar VB, Habuchi T, Grossman HB, Murphy WM, Hautmann SH, Hemstreet GP III, Bono AV, Getzenberg RH, Goebell P, Schmitz-Dräger BJ, et al: Bladder tumor markers beyond cytology: International consensus panel on bladder tumor markers. Urology 66: 35-63, 2005.

5. Kaymaz E, Ozer E, Unverdi H and Hucumenoglu S: Evaluation of MUC1 and P53 expressions in noninvasive papillary urothelial neoplasms of bladder, their relationship with tumor grade and role in the differential diagnosis. Indian J Pathol Microbiol 60: 510-514, 2017.

6. Wang Z, Wang X, Zhang D, Yu Y, Cai L and Zhang C: Long non-coding RNA urothelial carcinoma-associated 1 as a tumor biomarker for the diagnosis of urinary bladder cancer. Tumour Biol 39: 1010428317709990, 2017.

7. Peng F, Li H, Xiao H, Li L, Li Y and Wu Y: Identification of a three miRNA signature as a novel potential prognostic biomarker in patients with bladder cancer. Oncotarget 8: 105553-105560, 2017.

8. Duan R, Zhang Z, Zheng F, Wang L, Guo J, Zhang T, Dai X, Zhang S, Yang D, Kuang R, et al: Combining protein and mirna quantification for bladder cancer analysis. ACS Appl Mater Interfaces 9: 23420-23427, 2017.

9. Feng C, Sun P, Hu J, Feng H, Li M, Liu G, Pan Y, Feng Y, Xu Y, Feng $\mathrm{K}$ and Feng Y: miRNA-556-3p promotes human bladder cancer proliferation, migration and invasion by negatively regulating DAB2IP expression. Int J Oncol 50: 2101-2112, 2017.
10. Hirata H, Ueno K, Shahryari V, Tanaka Y, Tabatabai ZL, Hinoda Y and Dahiya R: Oncogenic miRNA-182-5p targets Smad4 and RECK in human bladder cancer. PLoS One 7: e51056, 2012.

11. Zhao X, He W, Li J, Huang S, Wan X, Luo H and Wu D: MiRNA-125b inhibits proliferation and migration by targeting SphK1 in bladder cancer. Am J Transl Res 7: 2346-2354, 2015.

12. Zeng T, Peng L, Chao C, Fu B, Wang G, Wang Y and Zhu X: miR-451 inhibits invasion and proliferation of bladder cancer by regulating EMT. Int J Clin Exp Pathol 7: 7653-7662, 2014.

13. Du M, Shi D, Yuan L, Li P, Chu H, Qin C, Yin C, Zhang Z and Wang M: Circulating miR-497 and miR-663b in plasma are potential novel biomarkers for bladder cancer. Sci Rep 5: 10437, 2015.

14. Alva A, Friedlander T, Clark M, Huebner T, Daignault S, Hussain M, Lee C, Hafez K, Hollenbeck B, Weizer A, et al: Circulating tumor cells as potential biomarkers in bladder cancer. J Urol 194: 790-798, 2015.

15. Scheffer AR, Holdenrieder S, Kristiansen G, von Ruecker A, Müller SC and Ellinger J: Circulating microRNAs in serum: Sovel biomarkers for patients with bladder cancer? World J Urol 32: 353-358, 2014.

16. Jin Q, Li XJ and Cao PG: miR-26b enhances radiosensitivity of hepatocellular carcinoma cells by targeting EphA2. Iran J Basic Med Sci 19: 851-857, 2016.

17. Gasque Schoof CR, Izzotti A, Jasiulionis MG and Vasques Ldos R: The roles of miR-26, miR-29 and miR-203 in the silencing of the epigenetic machinery during melanocyte transformation. Biomed Res Int 2015: 634749, 2015.

18. Yang F, Wang $\mathrm{H}$, Jiang $\mathrm{Z}, \mathrm{Hu} \mathrm{A}$, Chu L, Sun $\mathrm{Y}$ and Han J: MicroRNA19a mediates gastric carcinoma cell proliferation through the activation of nuclear factor- $\kappa \mathrm{B}$. Mol Med Rep 12: 5780-5786, 2015

19. Wadhwa N, Jatawa SK and Tiwari A: Republished: Non-invasive urine based tests for the detection of bladder cancer. Postgrad Med J 89: 352-357, 2013.

20. Wadhwa N, Jatawa SK and Tiwari A: Non-invasive urine based tests for the detection of bladder cancer. J Clin Pathol 65: 970-975, 2012.

21. Rossi R, Lichtner M, Iori F, Ermocida A, Mascia C, Mengoni F, Sauzullo I, Dini D, Mastroianni CM and Vullo V: Dendritic cells in blood and urine samples from bladder cancer patients undergoing BCG immunotherapy. Arch Ital Urol Androl 85: 157-163, 2013.

22. Blanca A, Cheng L, Montironi R, Moch H, Massari F, Fiorentino M, Raspollini MR, Scarpelli M and Lopez-Beltran A: Mirna expression in bladder cancer and their potential role in clinical practice. Curr Drug Metab 18: 712-722, 2017.

23. Blick C, Ramachandran A, McCormick R, Wigfield S, Cranston D, Catto J and Harris AL: Identification of a hypoxia-regulated miRNA signature in bladder cancer and a role for miR-145 in hypoxia-dependent apoptosis. Br J Cancer 113: 634-644, 2015.

24. Braicu C, Cojocneanu-Petric R, Chira S, Truta A, Floares A Petrut B, Achimas-Cadariu P and Berindan-Neagoe I: Clinical and pathological implications of miRNA in bladder cancer. Int J Nanomedicine 10: 791-800, 2015.

25. Cui X, Kong C, Zhu Y,Zeng Y,Zhang Z, Liu X, Zhan B, Piao C and Jiang Z: miR-130b, an onco-miRNA in bladder cancer, is directly regulated by NF- $\kappa \mathrm{B}$ and sustains NF- $\kappa \mathrm{B}$ activation by decreasing Cylindromatosis expression. Oncotarget 7: 48547-48561, 2016.

26. Egawa H, Jingushi K, Hirono T, Ueda Y, Kitae K, Nakata W, Fujita K, Uemura $\mathrm{M}$, Nonomura $\mathrm{N}$ and Tsujikawa $\mathrm{K}$ : The miR-130 family promotes cell migration and invasion in bladder cancer through FAK and Akt phosphorylation by regulating PTEN. Sci Rep 6: 20574, 2016.

27. Yang X, Cheng Y, Li P, Tao J, Deng X, Zhang X, Gu M, Lu Q and Yin C: A lentiviral sponge for miRNA-21 diminishes aerobic glycolysis in bladder cancer T24 cells via the PTEN/PI3K/AKT/mTOR axis. Tumour Biol 36: 383-391, 2015.

28. Mundhenk J, Hennenlotter J, Zug L, Alloussi SH, Todenhoefer T, Gakis G, Aufderklamm S, Scharpf M, Kuehs U, Stenzl A and Schwentner C: Evidence for PTEN-independent Akt activation and Akt-independent p27 (Kip1) expression in advanced bladder cancer. Oncol Lett 2: 1089-1093, 2011

29. Chang N, Lee HW, Lim JE, Jeong DE, Song HJ, Kim S, Nam DH, Sung HH, Jeong BC, Seo SI, et al: Establishment and antitumor effects of dasatinib and PKI-587 in BD-138T, a patient-derived muscle invasive bladder cancer preclinical platform with concomitant EGFR amplification and PTEN deletion. Oncotarget 7: 51626-51639, 2016.

This work is licensed under a Creative Commons Attribution-NonCommercial-NoDerivatives 4.0 International (CC BY-NC-ND 4.0) License. 